Check for updates

Cite this: RSC Adv., 2018, 8, 33059

Received 5th August 2018

Accepted 18th September 2018

DOI: $10.1039 / c 8 r a 06576 d$

rsc.li/rsc-advances

\section{Facile and template-free solvothermal synthesis of mesoporous/macroporous metal-organic framework nanosheets}

\author{
Boce Zhang, (D) *a Yaguang Luo, ${ }^{\text {b }}$ Kelsey Kanyuck, ${ }^{c}$ Natalie Saenz, ${ }^{\text {b } K e v i n ~ R e e d, ~}{ }^{a}$ \\ Peter Zavalij, ${ }^{c}$ Joseph Mowery ${ }^{\mathrm{b}}$ and Gary Bauchan ${ }^{\mathrm{b}}$
}

\begin{abstract}
A facile and template-free solvothermal method was developed as a bottom-up approach to synthesize mesoporous/macroporous MOF nanosheets in a simple and scalable way. It was found that starting coordination complexes of different copper(II)-ligand compounds mediated the controlled growth and morphology of MOF crystals. By controlling the size and shape of the MOF crystals, the possibility to adjust and tailor the structure and performances of the assemblies was demonstrated. This work provides a bottom-up approach to synthesize MOF films and nanosheets in a simple and scalable way, which may have potential in energy and biomedical applications.
\end{abstract}

\section{Introduction}

Metal-organic-frameworks (MOFs) are a class of compounds composed of metal ions or clusters within a network of organic ligand linkages. Similar to other porous materials, MOFs have attracted interest for use in gaseous or liquid adsorption, sensor materials, ${ }^{\mathbf{1}}$ catalysis, and biomedical devices. ${ }^{2,3}$ Large pore volumes and surface areas in conjunction with the well-organized structures that MOFs display set them apart from other porous materials such as zeolites and activated carbons. ${ }^{4}$ Furthermore, MOFs have enormous flexibility in composition and structure, can be synthesized quickly at low cost, and can be modified to have distinct functionalities. ${ }^{5}$

Theoretically, MOF pore size and functionality can be controlled by modification of metal ions and organic linkers. However, the conventional MOF synthesis limits the applicability as agglomeration decreases porosity and complicates gas adsorption and separation., ${ }^{\mathbf{4} 6}$ Recent work combined MOFs with other macroporous compounds to form materials with both micro- and macroporous features., ${ }^{7,8}$ Other methods avoid agglomeration and add porosity by arranging the MOFs into macroporous and/or mesoporous frameworks through packing into monoliths, dispersing in aerogels or arranging in films or membranes. ${ }^{2,3,9,10}$

MOF films and nanosheets, in particular, have been of interest for commercial applications, especially in molecular

${ }^{a}$ University of Massachusetts, Department of Biomedical and Nutritional Sciences, 833 Broadway St., Lowell, MA 01854, USA.E-mail: boce_zhang@uml.edu

${ }^{b}$ U.S. Department of Agriculture, Agricultural Research Service, 10300 Baltimore Ave., Beltsville, MD, 20705, USA. E-mail: yaguang.luo@ars.usda.gov

${ }^{c}$ University of Maryland, College Park, MD 20742, USA sieving, ${ }^{12,13}$ catalytic membranes and semiconductor materials $^{\mathbf{1 4}}$ due to their tendency to form mesopores and macropores. ${ }^{11}$ Other applications include liquid phase separations and chromatography as well as the storage, separation, or catalytic conversion of small molecules., ${ }^{\mathbf{9}, \mathbf{1 5}, \mathbf{1 6}}$ The applications of MOF nanofilms have even extended to photonic devices. ${ }^{17}$ However, the control of nanosheet thickness, crystallinity, and growth direction in MOF synthesis remains a work in progress. ${ }^{6,18,19}$

Many different methods have been employed to create MOF films and nanosheets. ${ }^{17,20-22}$ Most methods require templating MOFs onto other substances, including surfactant templating, ${ }^{23,24}$ liquid phase epitaxy ${ }^{25}$ among others., ${ }^{2,12,26}$ Though templating methods produce thin layers and homogeneous coatings, direct synthesis of template-free MOF nanosheets or films could significantly reduce the cost and complexity of synthesis, but this is largely unavailable.

Efficient, high-yielding methods that can produce MOF nanosheets with stable mesoporous frameworks are rare. ${ }^{\mathbf{1 9}}$ Synthesizing MOFs as freestanding and template-free nanosheets that retain morphological and structural integrity has been a challenge. ${ }^{6}$ To create films without templates, several groups have synthesized agglomerated nanosheets with organic solvents, followed by lengthy ultrasonication. ${ }^{\mathbf{1 4 , 1 9}}$ Recently, Li et al. developed a top-down delamination method to synthesize crystalline MOF nanosheets from bulk crystals. ${ }^{27}$ Rodenas et al. ${ }^{6}$ and Wu et al. ${ }^{17}$ employed a diffusion-mediated modulation of MOF molecular assembly to form a small layer within glass tubes. Nonetheless, applications are limited by the scalability issue of their synthetic methods.

In this study, a facile and template-free solvothermal method has been developed to synthesize mesoporous/macroporous 
MOF nanosheets. In our method, cupric salts (i.e., $\mathrm{Ac}^{-}, \mathrm{Cl}^{-}$, $\mathrm{NO}_{3}{ }^{-}, \mathrm{SO}_{4}{ }^{2-}$ ) and terephthalic acid (TPA) were dissolved in organic solvents containing dimethylformamide (DMF) and methanol $(\mathrm{MeOH})$. The entire synthesis was performed in a Teflon-lined solvothermal autoclave reactor. The growth rate and morphology of the MOFs were controlled using starting coordination complexes of different copper(II)-ligands.

\section{Experimental}

\section{Solvothermal synthesis of MOF}

Cupric salts, terephthalic acid (TPA), dimethylformamide (DMF), methanol and ethanol were purchased from Sigma Aldrich (St. Louis, MO, USA). The MOF matrices were synthesized via a modified solvothermal method. ${ }^{28}$ Cupric salt $(55 \mathrm{mg}$ of copper(II) chloride, $76 \mathrm{mg}$ of copper(II) nitrate, $74 \mathrm{mg}$ of copper(II) acetate, or $65 \mathrm{mg}$ of copper(II) sulfate) and $92 \mathrm{mg}$ of terephthalic acid (TPA) were dissolved in organic solvents containing $10 \mathrm{~mL}$ dimethylformamide (DMF) and $10 \mathrm{~mL}$ methanol or a solvent system inside a Teflon lined autoclave reactor (Col-Int Tech, Columbia, SC, USA). The solution was sonicated for 5 minutes to dissolve all particles and incubated for 24 hours at room temperature. The MOF product was synthesized by heating the reactor at either $120^{\circ} \mathrm{C}$ for 48 hours or $180{ }^{\circ} \mathrm{C}$ for 24 hours. The crystalline bluish solids were centrifuged $(5000 \times g$ for 10 minutes $)$ and washed three times with pure ethanol. The product was dried and activated in a vacuum oven at $100{ }^{\circ} \mathrm{C}$.

\section{X-ray diffraction crystallography (XRD)}

The MOF powder diffraction was characterized using a Bruker D8 Advance powder diffractometer (Bruker AXS Inc., Madison, WI, USA). The equipment was equipped with a $\mathrm{CuK} \alpha$ sealed tube (wavelength of $1.54178 \AA$ ), Ni $\beta$-filter, and positionsensitive LynxEye detector and operated in Bragg-Brentano mode ( $\theta-\theta$ geometry). Phase identification was performed using the international center for diffraction data (ICDD) powder diffraction database, and the data were further analyzed using Le Bail and Rietveld refinement on the supplier's TOPAS software (Bruker AXS Inc., Madison, WI, USA).

\section{Electron microscopy}

Scanning electron photomicrographs were captured using an S-4700 low-temperature field emission scanning electron microscope (LT-SEM) (Hitachi High Technologies America, Inc., Pleasanton, CA, USA) with a Quorum Cryo-Prep Chamber (Quorum Technologies, East Sussex, UK). All MOF samples were pretreated with a thin layer of Pt coating. All images were captured at $10 \mathrm{kV}$ accelerating voltage, and $10 \mathrm{~mm}$ working distance with a 4 pi Analysis System (Hillsborough, NC, USA). For transmission electron microscopy (TEM), ethanol solutions containing MOF samples were directly applied onto 400 mesh carbon-coated copper grids and allowed to absorb for 30 minutes, excess solution was wicked off and grids were air dried. Grids were imaged at 80
kV with a Hitachi HT-7700 TEM (Hitachi High Technologies America, Inc., Dallas, TX, USA).

\section{Characterization of MOF porosity and surface area}

Nitrogen $\left(\mathrm{N}_{2}\right)$ isotherms were measured on a Micromeritics TriStar II Plus (Micromeritics Instrument Co., Norcross, GA, USA) unit to full saturation, i.e., a relative pressure of approximately $\sim 1.0$ at $77 \mathrm{~K}$ to enable BET surface area and TOPV analyses. In addition, a low pressure $(P<1 \mathrm{~atm})$ ethylene isotherm was measured on a Micromeritics Accelerated Surface Area and Porosimetry System (ASAP, Norcross, GA, USA) unit at $25{ }^{\circ} \mathrm{C}$. The samples were activated on a Smart VacPrep degas unit by degassing in stages with a series of ramp/soak steps under dynamic vacuum till the final temperature reached $150{ }^{\circ} \mathrm{C}$ with a vacuum level of $<10^{-4}$ torr. Measurements of $\mathrm{N}_{2}$ BET on standard materials suggest accuracy to within approximately $5 \%$ at surface area values of $10 \mathrm{~m}^{2} \mathrm{~g}^{-1}$ and approximately $10 \%$ at levels of $\sim 0.5 \mathrm{~m}^{2} \mathrm{~g}^{-1}$ when at least $50 \mathrm{~m}^{2}$ of material are available for testing within the sample cell. Reproducibility for any given sample is dependent on the ability to regenerate the sample to the same degree of activation without modifying the surface or the pore structure. The cumulative volume was calculated based on a $\mathrm{BJH}$ analysis which relates pore size to relative pressure. In this analysis, a Halsey correction was used to account for monolayer thickness coverage.

\section{Results and discussion}

\section{Synthesis}

Fig. 1 shows the MOF morphologies resulting from starting coordination complexes of different copper(II)-ligands, including $\mathrm{Cu}^{2+}-\mathrm{Ac}^{-}, \mathrm{Cu}^{2+}-\mathrm{SO}_{4}{ }^{2-}, \mathrm{Cu}^{2+}-\mathrm{Cl}^{-}$, and $\mathrm{Cu}^{2+}-\mathrm{NO}_{3}{ }^{-}$. Fig. 2a highlights the TEM micrograph of the as-obtained MOF nanosheets mediated via the $\mathrm{Cu}^{2+}-\mathrm{Cl}^{-}$coordination complex. The free-standing nanosheets consisted of both individual films and macroporous agglomerated stacks. Fig. 2b shows an SEM micrograph of a cross-sectional view of the sponge-like MOF nanosheets in the agglomerated form. The thickness of an individual nanosheet was about $50 \mathrm{~nm}$, and the width of the nanosheet was over $15 \mu \mathrm{m}$ (Fig. 1a). Fig. 1e showed nanosheet structure at higher magnification power, and its selected area electron diffraction (SAED) pattern suggests that the nanosheet is polycrystalline. Similar structures were observed in the MOF synthesized via $\mathrm{Cu}^{2+}-\mathrm{NO}_{3}{ }^{-}$coordination complex (Fig. 1b), in which the MOFs had the form of porous and fragmented nanosheets. The high degree of crystallinity of both nanocubes and nanosheets produces clearly identifiable peaks in the X-ray powder diffraction (XRD) patterns.

\section{XRD}

Fig. 3 compares the XRD powders diffraction pattern between the anisotropic MOF nanosheet prepared from $\mathrm{Cu}^{2+}-\mathrm{Cl}^{-}$ coordination complexes and the MOF nanocubes formed via $\mathrm{Cu}^{2+}-\mathrm{Ac}^{-}$coordination complexes. The XRD analyses of the as-obtained MOFs with different morphologies revealed the 


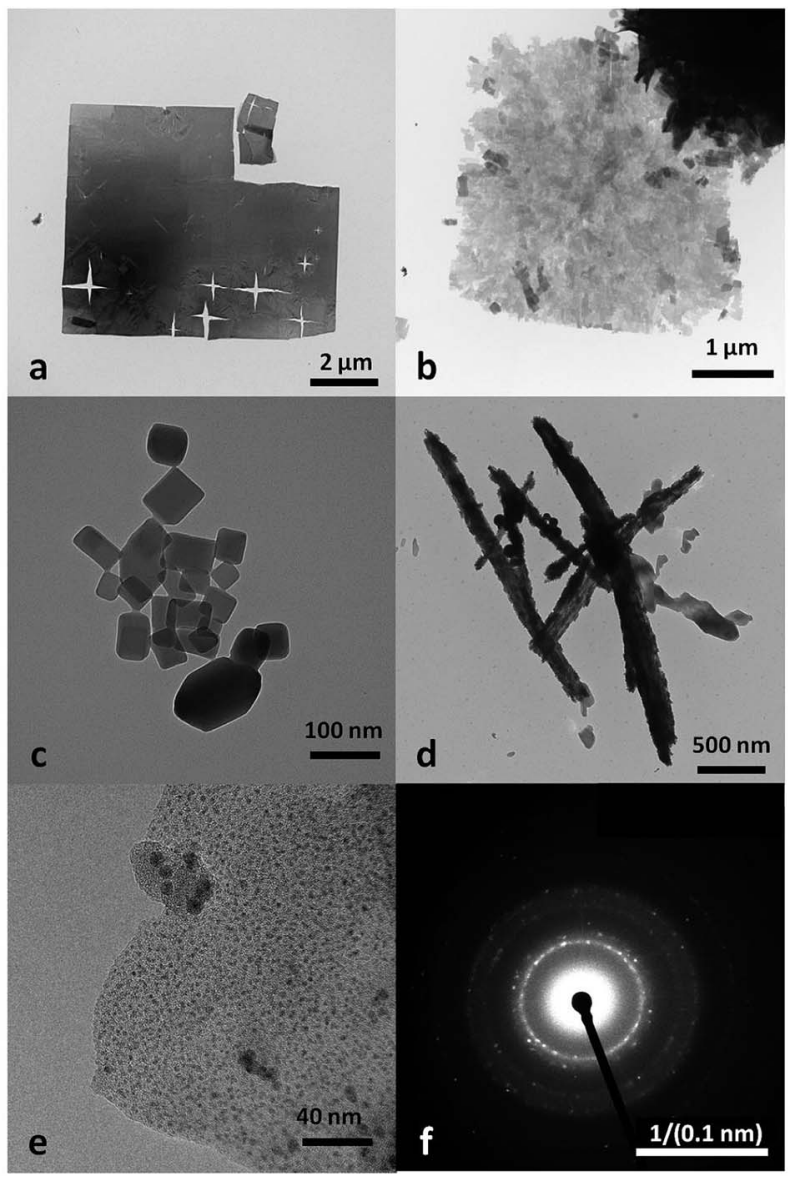

Fig. 1 TEM photomicrograph of MOF morphologies controlled via different copper(II)-ligand coordination complexes: (a) $\mathrm{Cu}^{2+}-\mathrm{Cl}^{-}$; (b) $\mathrm{Cu}^{2+}-\mathrm{NO}^{3-}$; (c) $\mathrm{Cu}^{2+}-\mathrm{Ac}^{-}$; (d) $\mathrm{Cu}^{2+}-\mathrm{SO}_{4}{ }^{2-}$; (e) $\mathrm{Cu}^{2+}-\mathrm{Cl}^{-}$at higher magnification; (f) selected area electron diffraction (SAED) pattern of $\mathrm{Cu}^{2+}-\mathrm{Cl}^{-}$nanosheet (a and e).

same cupric-TPA-based MOF. ${ }^{29}$ However, changes in the original cupric-ligand coordination complex could help engineer the size and shape of the MOF nanocrystals by determining the packing and growth rate of the MOF crystals during the solvothermal synthesis. The copper-ligand coordination complexes have different initial solubilities, in the order of $\mathrm{NO}^{3-}>\mathrm{SO}_{4}{ }^{2}>\mathrm{Cl}^{-}>\mathrm{Ac}^{-}$. It was found that lower
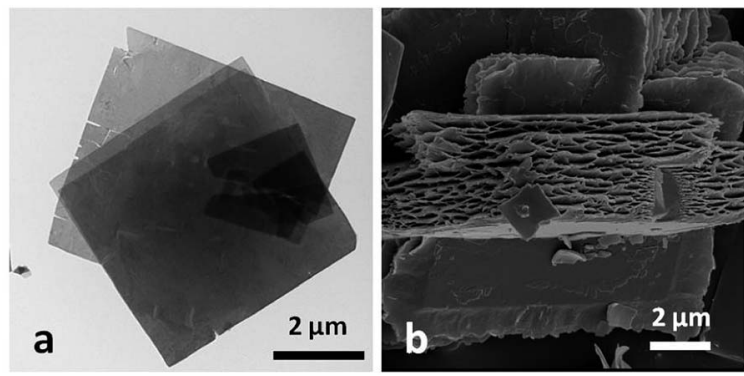

Fig. 2 (a) Micrograph of MOF nanosheet from transmission electron microscopy (TEM); (b) micrograph of MOF nanosheet from scanning electron microscopy (SEM).

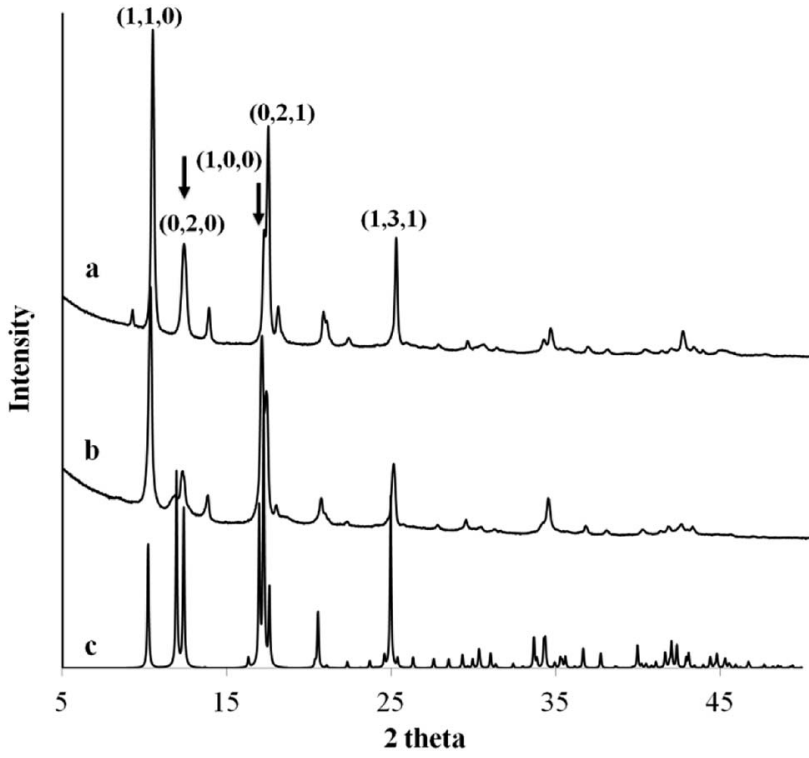

Fig. 3 The XRD powder diffraction pattern of MOF (a) nanocubes formed via $\mathrm{Cu}^{2+}-\mathrm{Ac}^{-}$coordination complex, (b) nanosheet formed via $\mathrm{Cu}^{2+}-\mathrm{Cl}^{-}$coordination complex, and (c) the identified single crystal diffraction pattern from ICDD database.

solubility with the $K_{\mathrm{sp}}$ below $1 \times 10^{-6}$ could restrain the growth of MOF crystals in sizes and in 2-dimension, and therefore, nanosheet were only obtained from $\mathrm{Cl}^{-}$and $\mathrm{Ac}^{-}$. In this study, the differences in crystal packing and gas storage capacity were investigated between two representative samples, which are MOF nanocubes $\left(\mathrm{Cu}^{2+}-\mathrm{Ac}^{-}\right)$and MOF nanosheets $\left(\mathrm{Cu}^{2+}-\mathrm{Cl}^{-}\right)$.

$\mathrm{Ac}^{-}$is a weaker ligand to $\mathrm{Cu}^{2+}$ and the organic ligand has better solubility in the organic solvent base of DMF and $\mathrm{MeOH}$ comparing to the $\mathrm{Cl}^{-}$ligand. The differences in ligand affinity and solubility could have led to a slower reaction rate and the anisotropy in the crystal growth kinetics. ${ }^{30}$ The MOF nanosheet revealed the significant change in the ratio of peak intensity (Fig. 3). The increase in peak intensity at $2 \theta=16.380^{\circ}$ was assigned to $(h, k, l=2,0,0)$ crystallographic plane, and the drop in peak intensity at $2 \theta=12.165^{\circ}$ was assigned to $(h, k, l=0,2,0)$ crystallographic plane. The significant change in (020 and 200) peak intensity (Fig. 3b) was a result of out-of-plane XRD
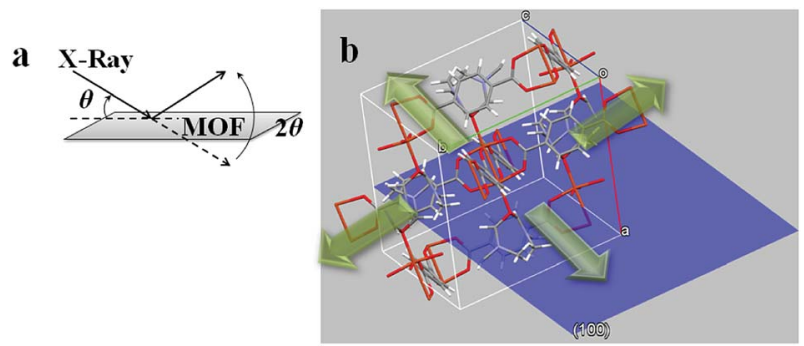

Fig. 4 (a) Illustration of out-of-plane XRD diffraction on MOF nanosheet; (b) hypothetical view of a MOF crystal unit with (100) plan (blue). 


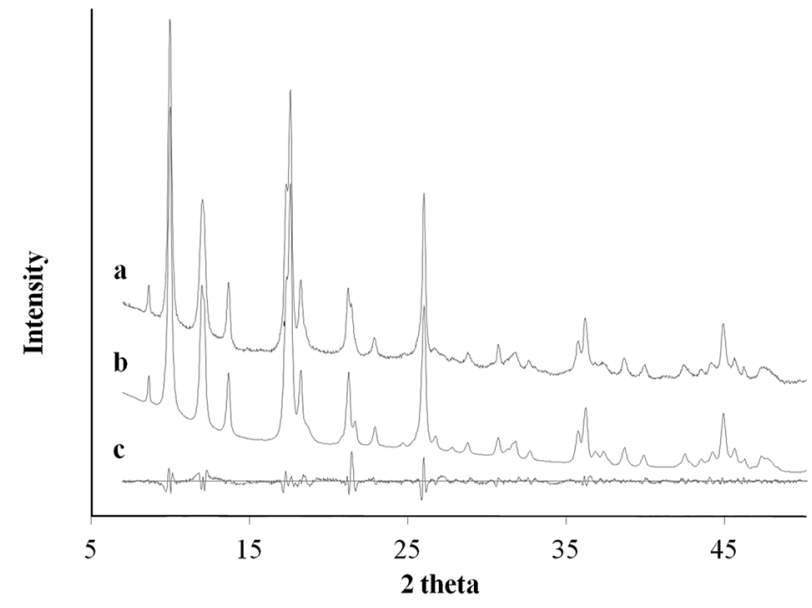

Fig. 5 XRD pattern profile of MOF nanocubes synthesized via $\mathrm{Cu}^{2+}-$ $\mathrm{Ac}^{-}$coordination complex (a); the calculated Le Bail refinement fitting (b); and the difference plot (c).

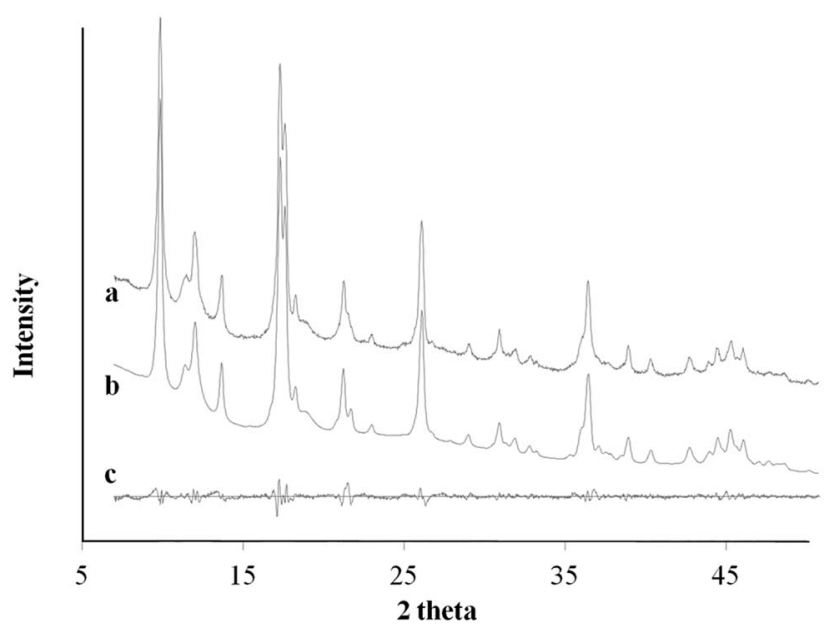

Fig. 6 XRD pattern profile of MOF nanosheets synthesized via $\mathrm{Cu}^{2+}{ }_{-}$ $\mathrm{Cl}^{-}$coordination complex (a); the calculated Le Bail refinement fitting (b); and the difference plot (c).

(Fig. 4a). The nanosheet pattern was further fitted using Le Bail refinement fitting for preferred orientation (PO) tendency analysis (Fig. 5 and 6). The results suggest that the stronger
$\mathrm{Cu}^{2+}-\mathrm{Cl}^{-}$coordination complex could either induce preferred covalent bond formation in the (100) plane along the $b, c$ axes or inhibit the crystallization process along the $a$ axis (Fig. 4b), in order to form the anisotropic MOF nanosheet. Nonetheless, the lattice parameters (Table 1), calculated from the Le Bail refinement of the XRD spectrums (Fig. 5 and 6), remained constant among MOF nanosheets, nanocubes and the International Centre for Diffraction Data (ICDD) database. ${ }^{28}$ Therefore, the staring coordination complexes of different copper(II)ligands determine the PO tendency toward crystallization of MOF in the solvothermal method without affecting unit cell structure.

\section{Porosity and functionalities}

The differences in crystal packing and MOF morphology also determine the porosity and functionality of the as-obtained MOF nanostructures. Fig. 7 shows the $\mathrm{N}_{2}$ isotherm, from which the Brunauer-Emmett-Teller (BET) surface area and total pore volume (TOPV). The sharp rise in the isotherms at low values of $P / P_{\mathrm{o}}$ is indicative of a large population of micropores $(<20 \AA)$. A comparison of the micropore volume to the total pore volume evaluated from the loading at $P / P_{\mathrm{o}}$ of $\sim 1.0$ reveals that $\sim 63 \%$ of the pore volume, of both samples, comes from the micropores (Fig. 8). This is also consistent with the high BET surface area values evaluated for these materials, i.e., 708 and $544 \mathrm{~m}^{2} \mathrm{~g}^{-1}$ for the MOF nanosheets and nanocubes, respectively. The cupric-TPA nanocubes have a similar BET surface area compared to a previous report, ${ }^{28}$ but the cupric-TPA nanosheet showed a $30.2 \%$ increase in BET surface area. The improvement of the surface area in MOF nanosheet could be attributed to the involvement of mesoporous and macroporous cavities (Fig. 2b), especially inside the agglomerated nanosheets structures. The cumulative and percent incremental pore volume plots are provided in Fig. 8a and $b$, respectively. The data suggests that a broad pore size distribution exists in the mesoporous and macroporous regions. The MOF nanocubes also have a small population of pores centered at $\sim 40 \AA$ and above $500 \AA$, whereas MOF nanosheets have an extensive amount of mesoporous and macroporous cavities above $400 \AA$.

Table 1 Le Bail refinement and lattice parameters of MOF nanocubes and nanosheets

\begin{tabular}{llll}
\hline Le Bail refinement & MOF nanocubes & MOF nanosheets & ICDD database \\
\hline$R$-value $\left(R_{\mathrm{wp}}\right)^{a}$ & 4.25 & 2.95 & N/A \\
$R$-value $\left(R_{\mathrm{p}}\right)^{a}$ & 2.78 & 2.10 & N/A \\
Specimen displacement & $-0.3858(16)$ & $-0.1848(17)$ & N/A \\
correction $(\mathrm{mm})$ & & & \\
Cell volume $\left(\AA^{3}\right)$ & $1211.11(18)$ & $1216.1(2)$ & $11.447(3)$ \\
$a(\AA)$ & $11.3862(9)$ & $14.482(5)$ & $11.4143(3)$ \\
$b(\AA)$ & $14.4948(12)$ & $7.757(2)$ & $14.2687(4)$ \\
$c(\AA)$ & $7.7670(6)$ & $108.92(2)$ & $7.7800(2)$ \\
$\beta\left({ }^{\circ}\right)$ & $109.126(6)$ & $108.119(1)$
\end{tabular}

${ }^{a}$ ' $R_{\mathrm{wp}}$ ' is $R$-value in weighted pattern; ' $R_{\mathrm{p}}$ ' is $R$-value in pattern; background subtracted and peak only contributions. 


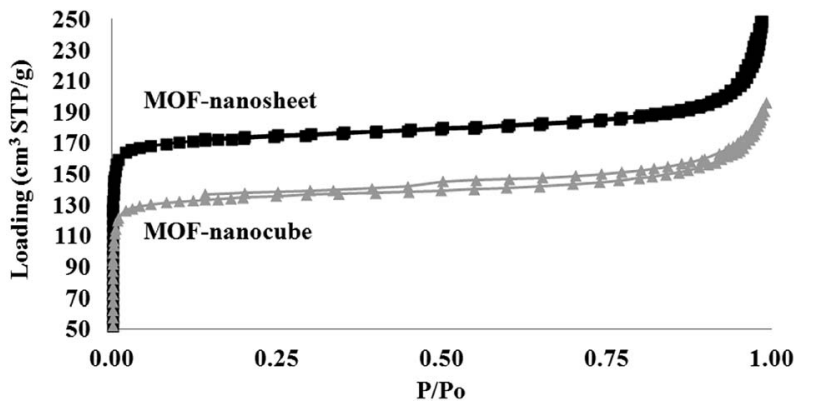

Fig. 7 Comparison of $\mathrm{N}_{2}$ adsorption-desorption isotherms measured at $77 \mathrm{~K}$ as a function of relative pressure between MOF nanosheet (top) and MOF nanocubes (bottom).

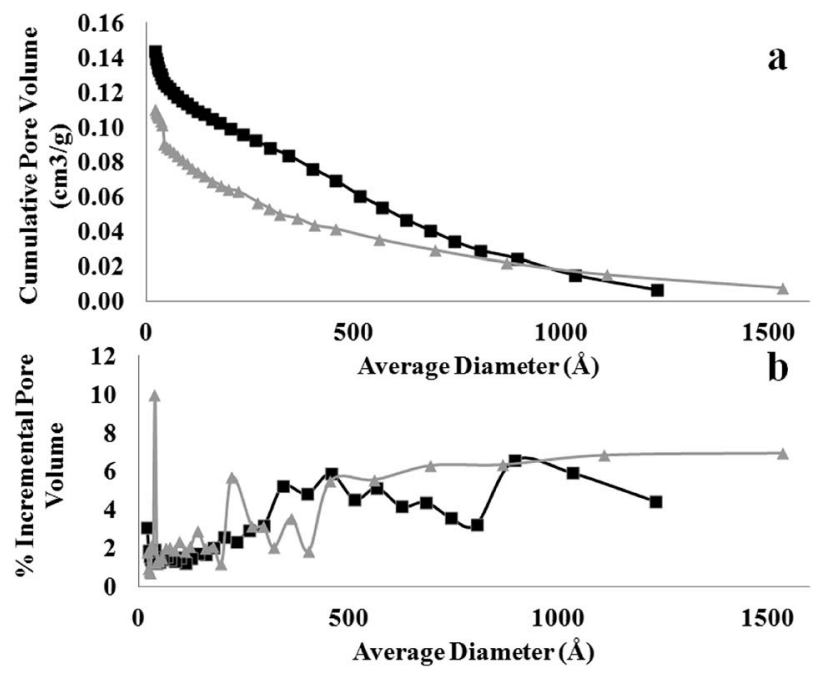

Fig. 8 Comparison of (a) cumulative and (b) incremental pore size distribution from desorption measured at $77 \mathrm{~K}$ between MOF nanosheet (square) and nanocube (triangle).

\section{Conclusions}

A facile and template-free solvothermal method has been developed for mesoporous and macroporous MOF nanosheets. It was found that starting coordination complexes of different copper(II)-ligand mediated the controlled growth and morphology of MOF crystals. By controlling the size and shape of the MOF crystals, it shows the possibility to adjust and tailor the structure and performances of the assemblies. This work provides a bottom-up approach to synthesize MOF films and nanosheets in a simple and scalable way, which may have potential applications for energy and biomedical applications.

\section{Conflicts of interest}

The authors declare no conflicting interests.

\section{Acknowledgements}

This work is supported by USDA-NIFA Specialty Crop Research Initiative Award No. 2016-51181-25403 and USDA-ARS Specific
Corporative Agreement No. S51600000035794. We acknowledge the support of Dr John Zielinski at Intertek Chemicals \& Pharmaceuticals service to conduct the work of $\mathrm{N}_{2}$ isotherms, BET and TOPV measurements. Mention of trade names or commercial products in this publication is solely for the purpose of providing specific information and does not imply recommendation or endorsement by the USDA; USDA is an equal opportunity provider and employer.

\section{References}

1 H. C. Streit, M. Adlung, O. Shekhah, X. Stammer, H. K. Arslan, O. Zybaylo, T. Ladnorg, H. Gliemann, M. Franzreb, C. Woell and C. Wickleder, ChemPhysChem, 2012, 13, 2699-2702.

2 A. Ahmed, M. Forster, R. Clowes, P. Myers and H. Zhang, Chem. Commun., 2014, 50, 14314-14316.

3 A. Ahmed, N. Hodgson, M. Barrow, R. Clowes, C. M. Robertson, A. Steiner, P. McKeown, D. Bradshaw, P. Myers and H. Zhang, J. Mater. Chem. A, 2014, 2, 9085-9090. 4 R. Adams, C. Carson, J. Ward, R. Tannenbaum and W. Koros, Microporous Mesoporous Mater., 2010, 131, 13-20.

5 J. Rowsell and O. M. Yaghi, Microporous Mesoporous Mater., 2004, 73, 3-14.

6 T. Rodenas, I. Luz, G. Prieto, B. Seoane, H. Miro, A. Corma, F. Kapteijn, F. X. Llabres I Xamena and J. Gascon, Nat. Mater., 2015, 14, 48-55.

7 Y. Zhang, X. Bo, C. Luhana, H. Wang, M. Li and L. Guo, Chem. Commun., 2013, 49, 6885-6887.

8 X. Wang, Q. Wang, Q. Wang, F. Gao, F. Gao, Y. Yang and H. Guo, ACS Appl. Mater. Interfaces, 2014, 6, 11573-11580.

9 M. R. Lohe, M. Rose and S. Kaskel, Chem. Commun., 2009, 6056-6058.

10 L. Li, S. Xiang, S. Cao, J. Zhang, G. Ouyang, L. Chen and C. Su, Nat. Commun., 2013, 4, 1774.

11 M. A. Green, Nat. Mater., 2010, 9, 539-540.

12 L. Peng, J. Zhang, Z. Xue, B. Han, X. Sang, C. Liu and G. Yang, Nat. Commun., 2014, 5, 4465.

13 Y. Liu, S. Liu and Z. Yue, $R S C A d v$., 2015, 5, 31742-31745.

14 A. Kondo, C. C. Tiew, F. Moriguchi and K. Maeda, Dalton Trans., 2013, 42, 15267-15270.

15 U. Mueller, M. Schubert, F. Teich, H. Puetter, K. SchierleArndt and J. Pastre, J. Mater. Chem., 2006, 16, 626-636.

16 K. A. Cychosz, A. G. Wong-Foy and A. J. Matzger, J. Am. Chem. Soc., 2009, 131, 14538-14543.

17 Y. Wu, F. Li, W. Zhu, J. Cui, C. Tao, C. Lin, P. M. Hannam and G. Li, Angew. Chem., Int. Ed., 2011, 50, 12518-12522.

18 R. Makiura, S. Motoyama, Y. Umemura, H. Yamanaka, O. Sakata and H. Kitagawa, Nat. Mater., 2010, 9, 565-571.

19 G. Xu, T. Yamada, K. Otsubo, S. Sakaida and H. Kitagawa, J. Am. Chem. Soc., 2012, 134, 16524-16527.

20 S. Hermes, M. K. Schroter, R. Schmid, L. Khodeir, M. Muhler, A. Tissler, R. W. Fischer and R. A. Fischer, Angew. Chem., Int. Ed., 2005, 44, 6237-6241.

21 E. Biemmi, C. Scherb and T. Bein, J. Am. Chem. Soc., 2007, 129, 8054-8055. 
22 C. Munuera, O. Shekhah, H. Wang, C. Woell and C. Ocal, Phys. Chem. Chem. Phys., 2008, 10, 7257-7261.

23 D. Bradshaw, S. El-Hankari and L. Lupica-Spagnolo, Chem. Soc. Rev., 2014, 43, 5431-5443.

24 L. Sun, J. Li, J. Park and H. Zhou, J. Am. Chem. Soc., 2012, 134, 126-129.

25 J. Liu, B. Lukose, O. Shekhah, H. K. Arslan, P. Weidler, H. Gliemann, S. Braese, S. Grosjean, A. Godt, X. Feng, K. Muellen, I.-B. Magdau, T. Heine and C. Woell, Sci. Rep., 2012, 2, 921.

26 Y. Hu, X. Dong, J. Nan, W. Jin, X. Ren, N. Xu and Y. M. Lee, Chem. Commun., 2011, 47, 737-739.
27 P. Li, Y. Maeda and Q. Xu, Chem. Commun., 2011, 47, 84368438.

28 C. G. Carson, K. Hardcastle, J. Schwartz, X. Liu, C. Hoffmann, R. A. Gerhardt and R. Tannenbaum, Eur. J. Inorg. Chem., 2009, 2338-2343.

29 C. G. Carson, K. Hardcastle, J. Schwartz, X. Liu, C. Hoffmann, R. A. Gerhardt and R. Tannenbaum, Eur. J. Inorg. Chem., 2009, 2338-2343.

30 Z. Zhuang, Q. Peng, B. Zhang and Y. Li, J. Am. Chem. Soc., 2008, 130, 10482-10483. 